\title{
Luiz Heitor and the studies of musical diversity in Brazil; a brief note
}

\author{
Samuel Araújo, UFRJ
}

After presenting his dissertation on the scales, rhythms and modes of native societies in Brazil, Luiz Heitor Correa de Azevedo became, in 1939, the first professor of the Musical Folklore chair at the National School of Music of the Universidade do Brasil, today's Federal University of Rio de Janeiro (UFRJ). Previously active in the field of opera and art music studies, he spent most of the year of 1941 working with Alan Lomax at the U.S. Library of Congress on folk music collecting methods and techniques, as a preparation for launching his own field research finally developed in the states of Goiás (1942), Ceará (1943), Minas Gerais (1944) and Rio Grande do Sul (1946), in an attempt to complement Mario de Andrade's similar efforts undertaken in 1938, also in collaboration with the Library of Congress (see also Zamith, 1992, 1991). This research consisted primarily of recordings made at his request, mostly in specially arranged performance situations seen as more suitable to facilitate the use of the intrusive equipment and obtain a better sound quality. This collection of over 300 recordings on disc, with separate catalogs for each state published by Azevedo, Henriqueta Rosa Fernandes Braga and Dulce Martins Lamas, his one-time students and chair successors, was housed at the Center for Folklore Research that he created at the same institution in 1943, and, since 2000, has been maintained by the Ethnomusicology Lab at UFRJ. So far, three master's theses (Aragão 2005, Mendonça 2007, Barros 2009) and two doctoral dissertations (Braga 2004, Prass 2009) have dealt with aspects of Azevedo's musical folklore research and archival work, in the two latter cases giving back copies of the original recordings to the recorded communities.

\section{References Cited}

ARAGÃo, Pedro de Moura. Luiz Heitor Corrêa de Azevedo e os Estudos de Folclore no Brasil: uma análise de sua trajetória na Escola Nacional de 
Música (1932-1947). Dissertação (Mestrado em Música)- Escola de Música, Universidade Federal do Rio de Janeiro, Rio de Janeiro. 2005.

BARROS, Felipe Santos de Lima. Construindo um acervo etnográficomusical; um estudo etnográfico sobre o arquivo de Luiz Heitor Correade Azevedo, seu método de campo e documentação produzida durante suasviagens a Goiás (1942), Ceará (1943) e Minas Gerais (1944).Dissertação (Mestrado em Música). Escola de Música da UniversidadeFederal do Rio de Janeiro. 2009.

BRAGA, Reginaldo Gil. Modernidade Religiosa entre Tamboreiros deNação: concepções e práticas musicas em uma tradição percussiva doextremo sul do Brasil. Porto Alegre, Tese (Doutorado emEtnomusicologia), Universidade Federal do Rio Grande do Sul. 2003.

MENDONÇA, Cecília de. A coleção Luiz Heitor Corrêa de Azevedo e osestudos de folclore no Brasil. Dissertação (Mestrado em MemóriaSocial). Programa de Pós-Graduação em Memória Social, UNIRIO, Rio deJaneiro. 2007.

PRASS, Luciana. Maçambiques, Quicumbis e Ensaios de Promessa: umre-estudo etnomusicológico entre quilombolas do sul do Brasil. Tese(Doutorado em Música). Universidade Federal do Rio Grande do Sul.2009.

ZAMITH, Rosa Maria Barbosa. A escola de Música da UFRJ e o estudo e pesquisa do folclore musical. Problemas e perspectivas. Anais do Simpósio Nacional de Ensino e Pesquisa de Folclore. São José dos Campos: Fundação Cultural Cassiano Ricardo,1992 p.135-145. Breve histórico do Centro de Pesquisas Folclóricas da UFRJ. Revista Brasileira de Música. Rio de Janeiro: UFRJ, 1991 no 19,p.228-236. 